\title{
Acute Calcium Pyrophosphate Crystal Arthritis Flare Rate and Risk Factors for Recurrence
}

\author{
Katherine A. Yates, Kazuki Yoshida, Chang Xu, Houchen Lyu, Vibeke Norvang, \\ Daniel H. Solomon, and Sara K. Tedeschi
}

ABSTRACT. Objective. Little is known about acute calcium pyrophosphate (CPP) crystal arthritis flare rates and risk factors for recurrence. We characterized flares and determined the rate and predictors of acute CPP crystal arthritis flares in an academic medical center cohort.

Methods. We performed a retrospective cohort study among a random sample of patients with acute CPP crystal arthritis identified in the Partners HealthCare electronic medical record, 1991-2017. Flare was defined as self-limited, acute-onset synovitis with synovial fluid CPP crystals and/or chondrocalcinosis, not better explained by another cause. We calculated incidence rates (IR) for acute CPP crystal arthritis flare among all subjects and by sex. We estimated HR for recurrent flare using univariate Cox models that accounted for within-person correlated data.

Results. We identified 70 patients with acute CPP crystal arthritis with a total of 111 flares. Recurrent flares occurred in $24 \%$ of patients; half of flares occurred in a previously unaffected joint. The acute CPP crystal arthritis flare rate was 11.4 per 100 person-years overall (95\% CI 8.2-15.4), 14.2 in women (95\% CI 9.6-0.1), and 7.1 in men (95\% CI 3.4-13.0). Cancer (HR 2.98, 95\% CI 1.33-6.68) and chronic kidney disease (HR 2.92, 95\% CI 1.10-7.76) were associated with a higher rate for recurrent flare.

Conclusion. Recurrent flares occurred in about one-fourth of patients with acute CPP crystal arthritis and often occurred in previously unaffected joints. The acute CPP crystal arthritis flare rate was twice as high in women as in men. (First Release May 15 2020; J Rheumatol 2020;47:1261-6; doi:10.3899/ jrheum.190778)

\section{Key Indexing Terms: PSEUDOGOUT \\ CALCIUM PYROPHOSPHATE CRYSTAL DEPOSITION ACUTE CALCIUM PYROPHOSPHATE CRYSTAL ARTHRITIS CRYSTAL ARTHROPATHY}

Acute calcium pyrophosphate (CPP) crystal arthritis, historically termed pseudogout, is the most dramatic manifestation of CPP crystal deposition (CPPD) disease ${ }^{1}$. Acute CPP crystal arthritis presents as acute mono- or oligoarticular

From the Department of Medicine, Vanderbilt University School of Medicine, Nashville, Tennessee; Division of Rheumatology, Inflammation and Immunity, Brigham and Women's Hospital; Department of Medicine, Harvard Medical School, Boston, Massachusetts, USA.

Supported by the US National Institutes of Health (grant numbers K23 AR075070 and P30 AR072577).

K.A. Yates, MD, Department of Medicine, Vanderbilt University School of Medicine; K. Yoshida, MD, ScD, Division of Rheumatology, Inflammation and Immunity, Brigham and Women's Hospital, and Department of Medicine, Harvard Medical School; C.Xu, MS, Division of Rheumatology, Inflammation and Immunity, Brigham and Women's Hospital; H. Lyu, MD, $P h D$, Division of Rheumatology, Inflammation and Immunity, Brigham and Women's Hospital; V. Norvang, MD, Division of Rheumatology, Inflammation and Immunity, Brigham and Women's Hospital; D.H. Solomon, MD, MPH, Division of Rheumatology, Inflammation and Immunity, Brigham and Women's Hospital, and Department of Medicine, Harvard Medical School; S.K. Tedeschi, MD, MPH, Division of Rheumatology, Inflammation and Immunity, Brigham and Women's Hospital, and Department of Medicine, Harvard Medical School.

Address correspondence to Dr. S.K. Tedeschi, Brigham and Women's Hospital, Division of Rheumatology, Inflammation and Immunity, 60 Fenwood Road, Suite 6016, Boston, Massachusetts 02115, USA.

E-mail: stedeschil@bwh.harvard.edu

Accepted for publication October 10, 2019. inflammatory arthritis, causing severe pain and functional limitation of the affected joints ${ }^{2}$. Some patients with acute CPP crystal arthritis experience recurrent flares, whereas others experience only 1 flare. A limited number of epidemiologic studies have focused on acute CPP crystal arthritis, despite its common occurrence ${ }^{3}$. The natural history of acute CPP crystal arthritis, including the rate of flare and patterns of joint involvement, remains incompletely understood.

Acute CPP crystal arthritis is thought to be more common in women than men. Hyperparathyroidism, osteoarthritis (OA), loop diuretic use, and bisphosphonate use were associated with acute CPP crystal arthritis in population-based case-control studies ${ }^{4,5}$.A large study drawing on US Veterans' Administration data used a broader definition of CPPD (i.e., not only acute CPP crystal arthritis) and identified some but not all of these associations ${ }^{6}$. Recurrent flares occurred in $\sim 20 \%$ of 106 patients with acute CPP crystal arthritis in a clinic-based cohort ${ }^{7}$. Baseline use of proton pump inhibitors, warfarin, and chemotherapy were each associated with risk for recurrent flare ${ }^{7}$. Whether time-varying risk factors are associated with the risk of future flare has not been evaluated. Acute CPP crystal arthritis flare rates, including sex-specific flare rates, remain unknown.

We aimed to estimate the rate of acute CPP crystal

Personal non-commercial use only. The Journal of Rheumatology Copyright @ 2020 . All rights reserved. 
arthritis recurrence, determine predictors of recurrence, and describe specific features of each flare.

\section{MATERIALS AND METHODS}

Study population and acute CPP crystal arthritis identification. Partners HealthCare is an academic medical center network in the greater Boston area including $>4$ million unique patients from 1991 to 2017 . We performed a retrospective cohort study among patients with acute CPP crystal arthritis identified in the Partners HealthCare electronic medical record (EMR), which includes data from inpatient care and outpatient visits. To identify our study sample, we randomly selected 1000 patients with at least 1 International Classification of Diseases, 9th ed (ICD-9) code for chondrocalcinosis $(712.1 \mathrm{x}, 712.2 \mathrm{x}, 712.3 \mathrm{x})$ or calcium metabolism disorder (275.49), or with 1 of 4 terms in narrative notes: (1) pseudogout, (2) CPPD, (3) calcium pyrophosphate, or (4) chondrocalcinosis. While ICD codes have low positive predictive value for identifying acute CPP crystal arthritis, we chose this sampling method given the lack of a published algorithm for identifying acute CPP crystal arthritis in EMR data $^{8}$. We performed detailed EMR review for the study definition of acute CPP crystal arthritis for all 1000 patients. The Partners HealthCare Institutional Review Board approved all aspects of this study (protocol 2016P001892).

Study definitions were developed based on historical diagnostic criteria for CPPD, European League Against Rheumatism 2011 terminology and diagnosis recommendations, and input from senior clinicians, because classification criteria for research do not currently exist ${ }^{1.9}$. "Definite" acute CPP crystal arthritis was defined as self-limited, acute-onset synovitis plus documentation of synovial fluid (SF) CPP crystals. "Probable" acute CPP crystal arthritis was defined as (1) self-limited, acute-onset synovitis in the wrist, knee, or ankle with chondrocalcinosis in that joint, and/or (2) self-limited, acute-onset synovitis with documentation by a rheumatologist or orthopedist that acute CPP crystal arthritis was the most likely diagnosis. Synovitis in the first metatarsophalangeal joint was not considered acute CPP crystal arthritis unless SF aspirated from that joint revealed CPP crystals, because gout is a more likely cause of synovitis at this site ${ }^{10}$.

Two authors (KAY and SKT) reviewed the EMR to determine whether each patient fulfilled the study definition of definite or probable acute CPP crystal arthritis. Clinical notes were searched for each of the 4 terms mentioned above (pseudogout, etc.) using an electronic text search tool that highlights EMR notes containing the term. If a term was present, we reviewed the notes in which it appeared. If none of the terms was present, we searched radiology reports for "chondrocalcinosis" and reviewed clinical notes around the time of that report. A board-certified rheumatologist (SKT) confirmed that all cases fulfilled the study definition.

Among all patients with acute $\mathrm{CPP}$ crystal arthritis, we identified recurrent flares by re-reviewing the EMR. We again reviewed all notes in which any of the 4 terms (pseudogout, etc.) or "swelling" appeared and determined whether the note was addressing a recurrent flare. Flares met the same criteria as the initial episode: (1) acute-onset synovitis plus documentation of SF CPP crystals, or (2) acute-onset synovitis in the wrist, knee, or ankle with chondrocalcinosis in that joint, and/or acute-onset synovitis and documentation by a rheumatologist or orthopedist that acute CPP crystal arthritis was the most likely diagnosis.

For the index episode and each recurrent flare, we recorded the date and collected data on joint(s) involved, whether the flare fulfilled the study definition of definite or probable acute CPP crystal arthritis, presence of chondrocalcinosis on imaging, whether joint aspiration was attempted, results of SF crystal analysis (if conducted), and treatment.

Baseline and followup time. The start of followup (index date) was the date of the visit or hospitalization during which acute CPP crystal arthritis was first diagnosed within our EMR system. Followup time was the time from the index date to recurrent flare or the last EMR note prior to December 31,2018 . We required a baseline period $\geq 30$ days before the index date and a followup period $\geq 30$ days following the index date and following each flare. To establish whether flares that were $<30$ days apart were truly separate flares, we required documentation that joint pain and swelling had resolved before recurring.

Covariates. Age was recorded at index date and updated at each recurrent flare; sex and race were recorded at index date. Baseline comorbidities were identified by reviewing data from the earliest EMR note through the index date. We used the EMR electronic text search tool to identify comorbidities that had been investigated as possible risk factors for acute CPP crystal arthritis in prior work and reviewed the pertinent notes to confirm presence of each condition. Comorbidities included cancer (active or in remission), coronary artery disease, stroke, hypothyroidism, hyperparathyroidism, chronic kidney disease (CKD), hemochromatosis, OA, gout, rheumatoid arthritis, and osteoporosis ${ }^{4.5}$.

Time-varying covariates were updated using data from the 30 days prior to recurrent flares; these included acute medical events and medications. We recorded acute medical events documented in the 30 days prior to index date or recurrent flare, including acute illness, trauma to the affected joint, and surgery. Acute illness was defined as a viral or bacterial illness with abrupt onset such as influenza, bronchitis, or pneumonia. We reviewed the medication list in the 30 days prior to index date or flare date for the presence of bisphosphonates, calcium supplements, thiazides, loop diuretics, proton pump inhibitors, warfarin, and chemotherapy based on prior literature associating these with increased risk for acute CPP crystal arthritis ${ }^{4,5,7}$.

Acute CPP crystal arthritis treatments were categorized as (1) no treatment or insufficient documentation about treatment, (2) nonsteroidal antiinflammatory drugs (NSAID) only, (3) colchicine with or without NSAID, and (4) oral or intraarticular steroid with or without colchicine or NSAID.

Statistical analysis. We summarized baseline characteristics among patients with and without recurrent acute CPP crystal arthritis flares using descriptive statistics. We calculated the flare incidence rate and 95\% CI overall and separately in men and women ${ }^{11}$. To estimate the rate for recurrent flare, we developed a series of Cox models accounting for within-person correlated data $^{12}$. We estimated univariate HR and $95 \%$ CI for recurrent flare for baseline comorbidities, characteristics of the index episode, time-varying acute medical events, and time-varying medications. The sample size limited our ability to fit a multivariable Cox model in a robust manner, so we focused on univariate models. Flare incidence rates and $95 \%$ CI were calculated using poisson.test in $\mathrm{R}$ version 3.4 .3 (cran.r-project.org). Descriptive statistics and Cox models were performed using SAS version 9.4 (SAS Institute).

\section{RESULTS}

We identified 70 patients with definite $(47.1 \%)$ or probable $(52.9 \%)$ acute CPP crystal arthritis based on the first episode, with a total of 111 acute CPP crystal arthritis episodes. Seventeen patients (24\%) had at least 1 recurrent flare, with a total of 41 recurrent flares. Baseline characteristics of patients with and without recurrent acute CPP crystal arthritis flares are presented in Table 1. Fifty-six percent of all patients were female; OA was the most common comorbidity. Half of the cohort received oral or intraarticular steroids with or without other treatments for the initial acute CPP crystal arthritis episode. Thirty percent received only NSAID, with the remainder receiving colchicine (with or without other treatments) or no treatment. Acute CPP crystal arthritis was diagnosed by rheumatologists $(43 \%)$, orthopedists $(24 \%)$, primary care or general medicine hospitalists $(20 \%)$, and other physicians (1\%). Acute CPP crystal arthritis most often occurred in the knee and wrist during the index episode (Table 2).

Thirteen patients had a history of gout in addition to

Personal non-commercial use only. The Journal of Rheumatology Copyright @ 2020 . All rights reserved. 
Table 1. Baseline characteristics in patients without and with recurrent pseudogout flares.

\begin{tabular}{|c|c|c|c|}
\hline Baseline Characteristics & $\begin{array}{l}\text { All Patients, } \\
\quad \mathrm{n}=70\end{array}$ & $\begin{array}{l}\text { No Recurrent Flare, } \\
\qquad \mathrm{n}=53\end{array}$ & $\begin{array}{l}\geq 1 \text { Recurrent Flare }, \\
\qquad \mathrm{n}=17\end{array}$ \\
\hline Age, yrs, mean (SD) & $72.3(14.2)$ & $72.1(14.8)$ & $72.8(12.8)$ \\
\hline Female & 55.7 & 52.8 & 64.7 \\
\hline White & 81.4 & 83.0 & 76.5 \\
\hline \multicolumn{4}{|l|}{ Basis for initial diagnosis } \\
\hline Synovitis + SF crystals* & 47.1 & 49.1 & 41.2 \\
\hline Synovitis + chondrocalcinosis & 52.9 & 50.9 & 58.8 \\
\hline \multicolumn{4}{|l|}{ Comorbidities } \\
\hline Cancer $^{+}$ & 22.9 & 20.8 & 29.4 \\
\hline Coronary artery disease & 20.0 & 20.8 & 17.7 \\
\hline Stroke & 4.3 & 3.8 & 5.9 \\
\hline Hypothyroidism & 20.0 & 22.6 & 11.8 \\
\hline Hyperparathyroidism & 1.4 & 1.9 & 0 \\
\hline CKD & 18.6 & 15.1 & 29.4 \\
\hline Hemochromatosis & 1.4 & 1.9 & 0 \\
\hline $\mathrm{OA}$ & 62.9 & 58.5 & 76.5 \\
\hline Gout & 18.6 & 18.9 & 17.7 \\
\hline RA & 2.9 & 3.8 & 0 \\
\hline Osteoporosis & 32.9 & 35.9 & 23.5 \\
\hline \multicolumn{4}{|c|}{ Medications 30 days prior to initial episode } \\
\hline Bisphosphonate & 5.7 & 7.6 & 0 \\
\hline Calcium & 27.1 & 28.3 & 23.5 \\
\hline Thiazide & 21.4 & 20.8 & 23.5 \\
\hline Loop diuretic & 22.9 & 26.4 & 11.8 \\
\hline Proton pump inhibitor & 35.7 & 32.1 & 47.1 \\
\hline Warfarin & 18.6 & 17.0 & 23.5 \\
\hline Chemotherapy & 2.9 & 1.9 & 5.9 \\
\hline \multicolumn{4}{|c|}{ Features of initial pseudogout episode } \\
\hline$>1$ joint involved & 11.4 & 9.4 & 17.7 \\
\hline Acute illness 30 days prior & 27.1 & 28.3 & 23.5 \\
\hline Joint trauma 30 days prior & 5.7 & 5.7 & 5.9 \\
\hline Postoperative 30 days prior & 10.0 & 9.4 & 11.8 \\
\hline \multicolumn{4}{|c|}{ Treatment of initial pseudogout episode } \\
\hline None or unclear** & 8.6 & 3.8 & 23.5 \\
\hline NSAID only & 30.0 & 35.9 & 11.8 \\
\hline Colchicine \pm NSAID & 10.0 & 11.3 & 5.9 \\
\hline Steroid ( \pm NSAID \pm colchicine) & 51.4 & 49.1 & 58.8 \\
\hline
\end{tabular}

Values are presented as percentages except where specified. * With or without chondrocalcinosis. ${ }^{+}$Cancers included lung, cervical, breast, prostate, thyroid, laryngeal, skin (non-melanoma), acute myelogenous leukemia, colon, bladder, endometrial, lymphoma. ** Either clear documentation of no treatment, or the medical record lacked clear documentation regarding whether treatment was given. SF: synovial fluid; CKD: chronic kidney disease; OA: osteoarthritis; RA: rheumatoid arthritis; NSAID: nonsteroidal antiinflammatory drugs.

fulfilling the study definition of acute CPP crystal arthritis: 1 had monosodium urate (MSU) crystals documented on a different occasion in a different joint, 1 had simultaneous observation of MSU and CPP crystals in the same joint, 7 had prior podagra, and 4 had been diagnosed with gout based on elevated uric acid and synovitis in a different joint. Recurrent flare characteristics. Of the 17 patients who experienced subsequent flares of acute CPP crystal arthritis, 7 patients experienced 1 recurrence, 5 patients experienced 2 recurrences, and 5 patients experienced 4 or more recurrences. Of the 41 recurrent flares, 20 involved previously unaffected joints (Table 2). Seven (17\%) recurrent flares were definite (crystal-proven) and $34(83 \%)$ were probable flares. The 34 probable flares occurred among 14 patients, 6 of whom (43\%) had an initial definite flare. Hands, wrists, knees, and ankles were the most common joints affected by recurrent flares. The interval between recurrent flares varied among individual patients and between patients (Table 2).

Of the 17 patients with recurrent flares, 3 had a history of gout. For the first patient, the recurrent flare was crystal-proven acute CPP crystal arthritis as well as crystal-proven gout; for the second patient, the recurrent flares occurred in a joint that had been initially diagnosed with crystal-proven acute CPP crystal arthritis; and for the third patient, the history of gout was podagra, the initial episode of CPP crystal arthritis was crystal-proven in the knee, and the recurrent flare occurred in the wrist (aspiration not attempted).

Incidence rate of recurrent acute CPP crystal arthritis flares.

Personal non-commercial use only. The Journal of Rheumatology Copyright @ 2020 . All rights reserved. 
Table 2. Joints affected by pseudogout among 17 patients with $\geq 1$ recurrent pseudogout flare.

\begin{tabular}{|c|c|c|c|}
\hline ID & Index Pseudogout Flare & Recurrent Flares & Since Prior Flare \\
\hline 1 & Right wrist & Right knee and right ankle & 39 \\
\hline \multirow[t]{4}{*}{2} & Left ankle & Right ankle & 7 \\
\hline & & Right knee & 35 \\
\hline & & Right ankle & 2 \\
\hline & & Right ankle & 6 \\
\hline \multirow[t]{4}{*}{3} & $\begin{array}{l}\text { Bilateral hands, bilateral } \\
\text { wrists, and right knee }\end{array}$ & Bilateral hands, bilateral wrists, right knee & 0.75 \\
\hline & & Bilateral hands & 2 \\
\hline & & Bilateral hands & 7 \\
\hline & & Left hand, left wrist, and left knee & 1 \\
\hline \multirow[t]{2}{*}{4} & Left wrist & Left shoulder & 2 \\
\hline & & Bilateral wrists and left knee & 9 \\
\hline 5 & Bilateral knees & Right talonavicular joint & 31 \\
\hline 6 & Left knee & Right wrist & 3 \\
\hline \multirow[t]{2}{*}{7} & Left wrist & Left wrist & 0.5 \\
\hline & & Left wrist & 1.5 \\
\hline 8 & Right elbow & Right elbow & 1 \\
\hline \multirow[t]{2}{*}{9} & Right knee & Right knee & 13 \\
\hline & & Right wrist & 54 \\
\hline 10 & Left knee & Left wrist & 8 \\
\hline \multirow[t]{7}{*}{11} & Left wrist & Right wrist & 1 \\
\hline & & Right knee & 4 \\
\hline & & Right knee & 1 \\
\hline & & Right knee & 0.75 \\
\hline & & Right knee & 2 \\
\hline & & Right wrist & 54 \\
\hline & & Right wrist & 0.5 \\
\hline 12 & Left ankle & Bilateral hands & 3 \\
\hline \multirow[t]{2}{*}{13} & Right knee & Left wrist & 111 \\
\hline & & Right shoulder & 5 \\
\hline \multirow[t]{4}{*}{14} & Left knee & Right knee & 6 \\
\hline & & Left knee and left ankle & 8 \\
\hline & & Right knee & 26 \\
\hline & & Right knee & 10 \\
\hline 15 & Right knee & Bilateral wrists & 79 \\
\hline \multirow[t]{5}{*}{16} & Left wrist and left elbow & Left wrist and left hand & 38 \\
\hline & & Left wrist & 11 \\
\hline & & Right wrist & 0.5 \\
\hline & & Bilateral wrists & 1.5 \\
\hline & & Bilateral wrists & 2 \\
\hline \multirow[t]{2}{*}{17} & Right wrist & Right wrist & 25 \\
\hline & & Left wrist & 9 \\
\hline
\end{tabular}

Recurrent flares are listed in the order in which they occurred.

We identified 41 recurrent flares among 70 patients with 361 person-years of followup. The acute CPP crystal arthritis flare rate was 11.4 (95\% CI 8.2-15.4) per 100 person-years among all patients. The flare rate in women $(14.2,95 \% \mathrm{CI}$ 9.6-20.1) per 100 person-years was twice as high as in men (7.1, 95\% CI 3.4-13.0) per 100 person-years. The median interval between recurrent flares was 5.7 months (range $0.4-110.5$ mos).

Predictors of acute CPP crystal arthritis recurrence. We identified an association between baseline comorbidities and risk for acute CPP crystal arthritis recurrence in univariate Cox models (Table 3). Patients with cancer or CKD had 3 times the risk for recurrence compared to subjects without those comorbidities (cancer HR 2.98, 95\% CI 1.33-6.68; CKD HR 2.92, 95\% CI 1.10-7.76). Patients who were treated with NSAID monotherapy at the initial episode had a lower risk for recurrence (HR $0.19,95 \%$ CI 0.04-0.90) than patients treated with steroids (with or without other treatments). Age, female sex, and acute medical events were not associated with risk for recurrent flare.

\section{DISCUSSION}

In this well-characterized cohort of 70 patients with definite or probable acute CPP crystal arthritis, one-fourth had a recurrent flare during 361 person-years of followup. Half of recurrent flares (49\%) involved a previously unaffected 
Table 3. Univariate HR (95\% CI) for risk of pseudogout recurrence.

\begin{tabular}{|c|c|}
\hline Variables & HR (95\% CI) \\
\hline $\operatorname{Age}^{\dagger}$ & $1.01(0.98-1.05)$ \\
\hline Female & $1.55(0.62-3.88)$ \\
\hline White & $0.79(0.29-2.19)$ \\
\hline \multicolumn{2}{|l|}{ Features of initial episode } \\
\hline Definite pseudogout & $0.74(0.33-1.67)$ \\
\hline$>1$ joint involved & $2.77(0.90-8.49)$ \\
\hline \multicolumn{2}{|l|}{ Treatment of initial episode } \\
\hline None or unclear* & $1.58(0.51-4.93)$ \\
\hline NSAID only & $0.19(0.04-0.90)$ \\
\hline Colchicine ( \pm NSAID) & $0.62(0.07-5.46)$ \\
\hline Steroid ( \pm NSAID \pm colchicine) & 1.00 (ref) \\
\hline \multicolumn{2}{|l|}{ Baseline comorbidities } \\
\hline Cancer & $2.98(1.33-6.68)$ \\
\hline Coronary artery disease & $1.59(0.58-4.37)$ \\
\hline Stroke & $0.83(0.14-4.88)$ \\
\hline Hypothyroidism & $0.53(0.15-1.83)$ \\
\hline Hyperparathyroidism & $0^{\ddagger}$ \\
\hline CKD & $2.92(1.10-7.76)$ \\
\hline Hemochromatosis & $0^{\ddagger}$ \\
\hline $\mathrm{OA}$ & $1.90(0.68-5.33)$ \\
\hline Gout & $0.91(0.29-2.85)$ \\
\hline RA & $0^{\ddagger}$ \\
\hline Osteoporosis & $0.98(0.39-2.45)$ \\
\hline \multicolumn{2}{|l|}{ Time-varying medications } \\
\hline Bisphosphonate & $1.02(0.25-4.08)$ \\
\hline Calcium & $1.54(0.68-3.47)$ \\
\hline Thiazide & $0.85(0.30-2.40)$ \\
\hline Loop diuretic & $0.51(0.13-1.96)$ \\
\hline Proton pump inhibitor & $1.81(0.74-4.42)$ \\
\hline Warfarin & $0.85(0.27-2.69)$ \\
\hline Chemotherapy & $1.95(0.17-22.50)$ \\
\hline \multicolumn{2}{|l|}{ Time-varying acute medical events } \\
\hline Acute illness & $0.97(0.39-2.44)$ \\
\hline Joint trauma & $0.82(0.09-7.51)$ \\
\hline Postoperative state & $0.59(0.16-2.17)$ \\
\hline
\end{tabular}

\footnotetext{
${ }^{\dagger}$ Age was treated as time-varying. * Either clear documentation of no treatment, or the medical record lacked clear documentation regarding whether treatment was given. ${ }^{\ddagger} \mathrm{HR}$ zero for baseline comorbidities that were absent in all patients with recurrent pseudogout flare. CKD: chronic kidney disease; OA: osteoarthritis; RA: rheumatoid arthritis; NSAID: nonsteroidal antiinflammatory drugs.
}

joint. The flare rate was 11.4 per 100 person-years overall, and was twice as high in women compared to men. We observed an increased risk for acute CPP crystal arthritis recurrence in patients with cancer and CKD.

The acute CPP crystal arthritis recurrence rate in our cohort was similar to the population-based gout recurrence rate in The Health Improvement Network (THIN) database (13.7 recurrent gout flares per 100 person-years) ${ }^{13}$. Whereas the rate of gout flares was higher in men than women in THIN, we identified a higher acute CPP crystal arthritis flare rate in women compared to men. In contrast to the study of gout flares in THIN, our acute CPP crystal arthritis sample size was much smaller and was not population-based and therefore results should be interpreted with caution. In a separate study using the THIN dataset, incident acute CPP crystal arthritis affected $0.18 \%$ of that population-based sample ${ }^{4}$. The percentage of patients with incident acute CPP crystal arthritis in our cohort was much higher (7\%), as expected, because our cohort included patients seen at an academic medical center with billing codes or relevant terms in notes.

The proportion of patients with at least 1 recurrent flare (24\%) and the median time to recurrence (5.7 mos) was similar to but slightly higher than reported in a previous retrospective study of 50 patients with acute CPP crystal arthritis (19\% with recurrent flare and 4.4 weeks in that study) by Lee, et al $l^{7}$. The authors observed an association between recurrent flare and use of proton pump inhibitors, warfarin, and chemotherapy, which we did not observe. Discrepancies in our findings may be due to differences in study population characteristics, including a different definition of acute CPP crystal arthritis. We excluded "possible" flares, which Lee, et al defined as acute arthritis of the knee, wrist, shoulder, hip, or ankle without requiring chondrocalcinosis and/or SF CPP crystals.

We observed that treatment of the initial acute CPP crystal arthritis episode differed between patients who did and did not have a recurrent episode. NSAID monotherapy was significantly associated with lower risk for flare recurrence compared to receiving steroids with or without other treatments. Confounding by indication may explain these findings; it is possible that patients with a more severe initial episode were treated with steroids and were also more likely to have a recurrent flare. Alternately, it is possible that the protective effect of NSAID monotherapy reflects lower prevalence of CKD in those patients, because CKD itself was associated with higher risk for recurrence.

CKD was associated with increased risk for acute CPP crystal arthritis in a large case-control study (OR 2.29, 95\% CI $1.30-4.01)^{4}$. In our current study, patients with CKD were at increased risk for recurrent flares (HR 2.92, 95\% CI 1.10-7.76). It is possible that CKD influences acute CPP crystal arthritis risk through secondary hyperparathyroidism, leading to altered calcium metabolism and subsequent formation and deposition of CPP crystals. CKD was defined as present if it was included on the problem list or clinical notes within 30 days of the index date and encompassed a range of severity, and it is possible that different stages of CKD may be associated with different risks for acute CPP crystal arthritis. Recurrent acute CPP crystal arthritis has been noted in case reports of patients with myelodysplastic syndrome (MDS) and/or acute myelogenous leukemia (AML) who have not received granulocyte colony stimulating factor (the latter of which has been linked with acute CPP crystal arthritis flares) $)^{14,15,16}$. The pathophysiology linking acute CPP crystal arthritis and MDS/AML remains unclear; it is possible that systemic inflammation related to neoplasm is a triggering factor. In our sample, 1 patient had AML and did have at least 1 recurrent flare. The majority of cancers in acute CPP crystal arthritis patients

Personal non-commercial use only. The Journal of Rheumatology Copyright $\subset$ 2020. All rights reserved. 
(with or without recurrent flares) were solid malignancies. It is possible that patients with cancer had more documented flares because of a greater number of healthcare visits, and thus more opportunities to document flares in the medical record, compared to patients without cancer.

Acute illness, joint trauma, and the postoperative state have been noted as triggers for acute CPP crystal arthritis in the literature ${ }^{17}$. In univariate models using time-varying covariates, we did not identify associations between recurrent flares and these 3 features. The lack of association may have been due to low prevalence of acute illness, joint trauma, and the postoperative state over time in our study population.

Our study had several limitations including small sample size; the absolute number of patients with at least 1 recurrent flare was 17. Additionally, only 7 recurrent flares (17\%) were crystal-proven, which may raise questions as to whether flares truly represented acute CPP crystal arthritis. It is possible that CPP and MSU crystals may have co-occurred in some patients, particularly those with a history of gout who did not undergo joint aspiration during the initial episode or recurrent flare ${ }^{18,19}$. Our data reflect routine clinical practice and provide a starting point for future analyses with prospective data collection from clinic-based cohorts of acute CPP crystal arthritis. The sample size enabled us to collect granular detail on each flare and its clinical context. The sample size limited our ability for multivariable analyses, so we could not evaluate the independent effect of each covariate adjusting for other covariates. Data were collected retrospectively by reviewing the EMR, so we were limited to the information documented as part of routine clinical care. This makes it likely that there was underascertainment of comorbidities including OA, medications (particularly over-the-counter NSAID), and recent illness. However, OA was more common among patients who fulfilled our stringent definition of acute CPP crystal arthritis (59\%) in an academic medical center cohort than in population-based acute CPP crystal arthritis cohorts $(35.6-46.3 \%)^{4,5}$. We applied a strict definition of acute CPP crystal arthritis that required documentation of synovitis; our recurrent flare rate may be an underestimate because we omitted episodes for which synovitis was not clearly described. Additionally, we were unable to record flares for which patients did not seek medical attention or received care in another medical center. Our cohort was composed of patients at a large academic medical center, and their comorbidities and treatments may not generalize to community-based populations.

We observed that recurrent flares occurred in nearly one-fourth of patients with acute CPP crystal arthritis. The acute CPP crystal arthritis flare rate was similar to a published gout flare rate, and was twice as high among women as men. Cancer and CKD were associated with greater risk for acute CPP crystal arthritis recurrence. Future work is critically needed to create standardized definitions of flares and to develop interventions to decrease the risk of acute CPP crystal arthritis recurrence.

\section{REFERENCES}

1. Zhang W, Doherty M, Bardin T, Barskova V, Guerne PA, Jansen TL, et al. European League Against Rheumatism recommendations for calcium pyrophosphate deposition. Part I: terminology and diagnosis. Ann Rheum Dis 2011;70:563-70.

2. Rosales-Alexander JL, Balsalobre Aznar J, Magro-Checa C. Calcium pyrophosphate crystal deposition disease: diagnosis and treatment. Open Access Rheumatol 2014;6:39-47.

3. Abhishek A, Neogi T, Choi H, Doherty M, Rosenthal AK, Terkeltaub R. Review: Unmet needs and the path forward in joint disease associated with calcium pyrophosphate crystal deposition. Arthritis Rheumatol 2018;70:1182-91.

4. Rho YH, Zhu Y, Zhang Y, Reginato AM, Choi HK. Risk factors for pseudogout in the general population. Rheumatology 2012; 51:2070-4.

5. Roddy E, Muller S, Paskins Z, Hider SL, Blagojevic-Bucknall M, Mallen CD. Incident acute pseudogout and prior bisphosphonate use: Matched case-control study in the UK-Clinical Practice Research Datalink. Medicine 2017;96:e6177.

6. Kleiber Balderrama C, Rosenthal AK, Lans D, Singh JA, Bartels CM. Calcium pyrophosphate deposition disease and associated medical comorbidities: a national cross-sectional study of US veterans. Arthritis Care Res 2017;69:1400-6.

7. Lee JS, Hong S, Kwon OC, Kim YG, Lee CK, Yoo B. Clinical features and risk of recurrence of acute calcium pyrophosphate crystal arthritis. Clin Exp Rheumatol 2019;37:254-9.

8. Tedeschi SK, Solomon DH, Liao KP. Pseudogout among patients fulfilling a billing code algorithm for calcium pyrophosphate deposition disease. Rheumatol Int 2018;38:1083-8.

9. McCarty DJ. Calcium pyrophosphate dihydrate crystal deposition disease: nomenclature and diagnostic criteria. Ann Intern Med 1977;87:241-2.

10. Huskisson EC, Balme HW. Pseudopodagra. Differential diagnosis of gout. Lancet 1972;2:269-71.

11. Collett D. Modelling binary data. Boca Raton, FL: Chapman and Hall/CRC; 1999.

12. Andersen PK, Gill RD. Cox's regression model for counting processes: a large sample study. Ann Stat 1982;10:1100-20.

13. Rothenbacher D, Primatesta P, Ferreira A, Cea-Soriano L, Rodriguez LA. Frequency and risk factors of gout flares in a large population-based cohort of incident gout. Rheumatology 2011;50:973-81

14. Tedeschi SK, Stone RM, Helfgott SM. Calcium pyrophosphate crystal inflammatory arthritis (pseudogout) with myelodysplastic syndrome: A new paraneoplastic syndrome? J Rheumatol 2017;44:1101-2.

15. Iqbal SM, Aslam HM, Faizee F, Qadir S, Waheed S. Pseudogout: an autoimmune paraneoplastic manifestation of myelodysplastic syndrome. Cureus 2018;10:e3372.

16. Slostad JA, Wild EM, Anderson CM, Ingram C. Intractable neck pain in a patient with newly diagnosed AML: An underrecognized cause of a treatable syndrome. J Pain Symptom Manage 2019;57:e3-e5.

17. Rosenthal AK, Ryan LM. Calcium pyrophosphate deposition disease. N Engl J Med 2016;374:2575-84.

18. Malik A, Schumacher HR, Dinnella JE, Clayburne GM. Clinical diagnostic criteria for gout: comparison with the gold standard of synovial fluid crystal analysis. J Clin Rheumatol 2009;15:22-4.

19. Robier C, Neubauer M, Quehenberger F, Rainer F. Coincidence of calcium pyrophosphate and monosodium urate crystals in the synovial fluid of patients with gout determined by the cytocentrifugation technique. Ann Rheum Dis 2011;70:1163-4.

Personal non-commercial use only. The Journal of Rheumatology Copyright (c) 2020. All rights reserved. 